\title{
Sleep disturbances in diabetic peripheral neuropathy patients: a clinical and polysomnographic study
}

\author{
Wafik Said Bahnasy ${ }^{1,3^{*}} \mathbb{D}$, Yasser Abo Elfotoh El-Heneedy ${ }^{1}$, Ehab Ahmed Shawky El-Seidy ${ }^{1}$, Nema Ata Allah Labib ${ }^{1}$
} and Ibrahim Salah Eldeen Ibrahim²

\begin{abstract}
Background: Disordered sleep breathing is a common complication of diabetic peripheral neuropathy (DPN) manifested by excessive daytime sleepiness, morning headache, morning dizziness, cognitive decline, and mood changes.

Methods: This study was performed on 30 non-obese type 2 diabetic patients; 20 with clinically evident DPN and 10 without. Ten age-, sex-, and body mass index-matched healthy control subjects were also included. Patients and control were subjected to history taking, neurological examination, glycated hemoglobin, and clinical assessment of the sensori-motor manifestations by the neuropathy symptom score and neuropathy disability score. The autonomic nervous system was evaluated clinically by the systolic blood pressure response to standing and heart rate response to each of standing, Valsalva, and deep breath. Finally, sleep was assessed by one-night polysomnogram (PSG) followed by multiple sleep latency test in the next day.

Results: The study showed significant increase in sleep apnea syndromes in diabetic peripheral neuropathy patients compared to diabetic neuropathy free patients and healthy control $(p<0.0001)$. The sleep apnea was mainly obstructive and to a little extent mixed (obstructive/central) sleep apnea. The severity of sleep PSG abnormalities was positively correlated with the severities of sensory, motor, and autonomic manifestations.

Conclusions: Non-obese type 2 diabetic patients complicated by peripheral neuropathy especially those having dysautonomia are at increased risk of developing sleep disordered breathing resulting in their excessive daytime sleepiness, decreased productivity, and poor glycemic control.
\end{abstract}

Keywords: Diabetic peripheral neuropathy, Diabetic autonomic neuropathy, Sleep apnea syndrome, Multiple sleep latency test and polysomnography

\section{Background}

Diabetic peripheral neuropathy (DPN) is one of the most common complications of diabetes (DM) affecting 20$60 \%$ of type 2 diabetic patients ( $\mathrm{Qu}$ et al. 2017). It is defined as somatic and/or autonomic neuropathy attributed solely to DM. Diabetic autonomic neuropathy (DAN) is the most under-diagnosed, yet one of the most serious complications of DM caused by damage of

\footnotetext{
* Correspondence: wafiq.elbahnasi@med.tanta.edu.eg

${ }^{1}$ Department of Neuropsychiatry, Tanta University, Tanta 31527, Egypt

${ }^{3}$ Department of Neuropsychiatry, Faculty of Medicine, Tanta University, Tanta

31511, Egypt

Full list of author information is available at the end of the article
}

efferent parasympathetic and/or sympathetic nerves (Feldman et al. 2017). Diabetic autonomic neuropathy is usually of insidious onset in long standing poorly controlled diabetics (Villafaina et al. 2017). Its most common manifestations include resting tachycardia, postural hypotension, arrhythmias, gastroparesis, diarrhea, constipation, gustatory sweating, dry skin, erectile dysfunction, retrograde ejaculation, atonic bladder, abnormal renal sodium handling, pupillary dysfunction, and bronchoconstriction (Martin et al. 2014).

There is a reciprocal relationship between sleep apnea syndromes (SAS) and DM while the presence of DAN increases this negative interaction. Patients with DAN 
have nocturnal symptoms interfering with their sleep quality and are at higher risks of sudden death during sleep up to fivefolds than those without (Pop-Busui et al. 2010). On the other hand, SAS in diabetics result in increased insulin resistance, impaired glucose tolerance, and excessive daytime sleepiness (EDS) which results in patients' easy fatigue, cognitive decline, mood changes, and reduced productivity (Schober et al. 2011).

\section{Aim of the work}

Was to study the possible existence of sleep abnormalities in DPN patients and their relations to the severities of both sensori-motor and autonomic manifestations.

\section{Methods}

The present study was conducted on 30 non-obese type 2 diabetic patients attending the neurology and internal medicine diabetes clinics, Tanta University Hospitals, in the period between 1st of April and 1st of December 2015. Patients were divided into two groups; group I included 20 clinically evident sensori-motor DPN patients with or without autonomic manifestations and group II included 10 diabetic neuropathies free (DNF) patients. The study also included 10 healthy control subjects (group III) matching the patients' age, sex, and body mass index (BMI).

Exclusion criteria invloved patients with respiratory or cardiac problems, chronic pain, and advanced metabolic, neuropsychiatric or endocrinal disorders affecting sleep and patients with $\mathrm{BMI}>28$ and history of medications intake affecting sleep and who are heavy smokers or drug abusers.

The protocol of the study was approved by The Research Ethics Committee and Quality Assurance Unit, Faculty of Medicine, Tanta University. Participation was voluntary, all participants received detailed information concerning the aims of the study and the possible risks, and an informed consent was obtained from all prior to the commencement in the study.

Diabetes mellitus was diagnosed according to American Diabetes Association 2015 (Polonsky and Burant 2016). Patients and controls were submitted to history taking, neurological examination, and routine laboratory investigations. Clinical diagnosis of DPN and grading of its severity was done using the neuropathy symptom score (NSS) and neuropathy disability score (NDS). A total NSS of 3-4 considered mild, 5-6 moderate, and 7-9 severe symptoms. A total NDS of 3-5 considered mild, 6-8 moderate, and 9-10 severe disability. Neuropathy was diagnosed in patients with moderate disability with or without symptoms, or mild disability with moderate symptoms. Mild disability and/or mild symptoms were not considered adequate to diagnose clinical evident DPN (Kisozi et al. 2017; Cabezas-Cerrato 1998). Dysautonomia was clinically assessed by measuring systolic blood pressure response to standing and heart rate response to each of standing, Valsalva, and deep breath.

All subjects were submitted to one-night polysomnogram (PSG) followed in the next day by multiple sleep latency test (MSLT) which is an objective assessment of EDS (Littner et al. 2005). PSG was performed by a Somon Medics Gmbh (Am SonnenstuhL63, D-97236 Rander Sacker, Germany, Type: SOMNO screen ${ }^{\text {twp }}$ plus, SN: 4259, kw45: 2014). Each PSG included EEG channels montages (O1/A2, C3/A2, C4/A1 and O2/A1), electrooculography (LOC-A1/A2 and ROC-A1/A2), surface tibial and submental EMG, and modified V2 lead ECG. For respiratory sensors, nasal and oral signals by thermal airflow sensors (thermistor) were used, tracheal sounds microphone was applied, and the chest and abdominal effort was measured by dual thoracoabdominal RIP (respiratory inductance plethysmography) belts.

The studied parameters were scored according to The American Academy of Sleep Medicine Scoring Manual, 2012 (Grigg-Damberger 2012). Sleep latency (SL) refers to the length of time taken in transition from wakefulness to sleep, wake after sleep onset (WASO) is the minutes of wake after sleep onset but before the final awakening, sleep efficiency (SE) is the total sleep time (TST) divided by the total in bedtime, sleep fragmentation (SF) is the number of sleep cycles/night, and sleep stage transition index (SSTI) is the number of transition between various sleep stages/hour. The pulse transit time (PTT) is the time taken for the arterial pulse wave to travel from the aortic valve to a peripheral site which reflects the intrathoracic pressure. An arousal refers to abrupt shift of EEG activities which last for $\geq 3 \mathrm{~s}$ and are preceded by $\geq 10$ s of sleep. An apnea was defined as $\geq 90 \%$ drop in the thermistor excursion signal of $\geq 10 \mathrm{~s}$ and hypopnea which was defined as a decrease in thermistor signal by $>30 \%$ for $>10 \mathrm{~s}$ accompanied by a decrease of oxyhemoglobin saturation $\geq 4 \%$. The patient was considered to have sleep apnea syndrome (SAS) if the apnea hypopnea index (AHI) is $\geq 15$ or if the AHI $\geq 5$ associated with insomnia, cardiovascular comorbidities, or diurnal symptoms mainly EDS, impaired cognition, or mood changes (Sateia 2014).

Statistical analysis was conducted using SPSS version 19 (Statistical Package for Social Studies) created by IBM, Chicago, IL, USA. For numerical values, the range and mean \pm standard deviations were calculated. For categorical variable, the number and percentage were calculated and differences between subcategories were tested using the $z$-score test, ANOVA, Tukey's tests, and post-hoc tests. Correlation analysis was performed using Pearson's correlation test. $p$ value $<0.05$ was considered statistically significant. 


\section{Results}

The study included 20 DPN patients, with mean ages $48.25 \pm 7.7$ years, mean BMI $25.7 \pm 1.97 \mathrm{~kg} / \mathrm{m}^{2}, 12$ females (60\%) and 8 males (40\%). Groups II and III were selected to be age, sex and BMI matched with DPN group. Diabetes duration (years) and glycated hemoglobin \% were significantly higher in DPN group than DNF group with $p$ value $=0.0003$ and $<0.0001$ respectively (Table 1 ) Diabetes treatment regimens in DPN patients were changeable and cannot be correlated with sleep changes, and at the time of research, 10 patients were on different types of oral hypoglycemic drugs, 4 on insulin, and 6 on combination therapy.

History and examinations of DPN group revealed 9 patients (45\%) had slippering of slippers, 8 patients (40\%) had postural dizziness, and 11 patients (55\%) had urinary symptoms. Five male patients (25\%) had erectile dysfunctions, 1 patient (5\%) had history of diabetic ophthalmoplegia, 4 patients (20\%) had gustatory sweating, 5 patients (25\%) had peripheral limb ischemia, and 7 patients (35\%) had gastrointestinal symptoms mainly bloating, gastric distension, and constipation alternating with diarrhea.

Neuropathy symptom score among DPN group was $7.1 \pm 1.48$ compared to $2 \pm 1.05$ and $1.6 \pm 0.69$ in DNF and control groups respectively with $p<0.0001$. Moreover, NDS in DPN group were $8.05 \pm 1.5$ compared to $2.1 \pm 0.99$ and $1.5 \pm 0.52$ in DNF and control groups respectively with $p<0.0001$ (Table 1 ).

Thirteen DPN patients (65\%) had objective autonomic manifestation in one of the included examinations. The study showed that, postural systolic hypotension, postural tachycardia, abnormal heart rate response to deep breath were significantly higher in DPN group compared to DNF and control groups. Heart rate response to Valsalva showed non-significant difference between the three studied groups (Table 1). The degree of dysautonomia was directly proportional with disease duration,
HBA1c \%, and severity of sensori-motor manifestations measured by each of NSS and NDS.

Regarding sleep architecture, 8 DPN patients (40\%) had SAS which was significantly higher than each of DNF and control groups (10\% in each). In the DPN group, 6 patients had obstructive sleep apnea (OSA) and 2 had mixed sleep apnea (MSA) while the SAS subjects in groups 2 and 3 were OSA only. In a parallel way, the AHI was significantly higher in group I than groups 2 and 3 with $p$ value $<0.0001$ (Table 2 ).

The mean latencies of MSLT were significantly diminished in DPN group (9.2 $\pm 4.03 \mathrm{~min})$ compared to DNF and control groups $(15.1 \pm 3.44$ and $16.3 \pm 2.2$ in respectively) with $p$ value $<0.0001$. Sleep microstructure showed significant decrease in each of sleep latencies, sleep efficiencies, pulse transit time, lowest $\mathrm{O}_{2}$ saturations and REM\% of total sleep time (TST) in DPN group compared to DNF and control groups. At the same time, DPN patients showed significant increase in sleep fragmentations, snore \% of TST and desaturation, arousal, snore and periodic limb movement indices compared to DNF and control groups (Table 2 and Fig. 1).

The mean latencies of MSLT in DPN patients showed significant negative correlations with each of diabetes duration, HBA1c, NSS, NDS, postural systolic hypotension, and postural tachycardia. On the other hand, the severity of sleep apnea measured by AHI in DPN patients showed significant positive correlations with each of disease duration, HBA1c, NSS, NDS, postural systolic hypotension, and postural tachycardia (Table 3, Fig. 2).

\section{Discussion}

Sleep apnea syndrome is a disorder in which the airflow suddenly stops or diminishes by $>50 \%$ during sleep leading to frequent nocturnal hypoxia and arousals which significantly impairs the sleep quantity and/or quality with consequent deleterious daytime effects including EDS, resistant hypertension, and ischemic heart diseases

Table 1 Glycated hemoglobin, neuropathy symptom and disability scores, and autonomic nervous system assessment among diabetic neuropathy patients (group I), diabetic patients without neuropathy (group II) and healthy control (group III)

\begin{tabular}{|c|c|c|c|c|c|c|c|c|}
\hline & \multicolumn{2}{|l|}{ Group I } & \multicolumn{2}{|l|}{ Group II } & \multicolumn{2}{|c|}{ Group III } & \multicolumn{2}{|l|}{ ANOVA } \\
\hline & Range & Mean \pm SD & Range & Mean \pm SD & Range & Mean \pm SD & $f$ value & $p$ value \\
\hline HBA1C (\%) & $6.7-11.5$ & $8.33 \pm 1.48$ & $6.8-8.5$ & $7.57 \pm 0.53$ & $5.5-6.3$ & $5.90 \pm 0.26$ & 16.18 & $<0.0001^{*}$ \\
\hline NSS & $5-9$ & $7.1 \pm 1.48$ & $1-4$ & $2 \pm 1.05$ & $1-3$ & $1.6 \pm 0.69$ & 92.73 & $<0.0001^{*}$ \\
\hline NDS & $6-10$ & $8.05 \pm 1.5$ & $1-4$ & $2.1 \pm 0.99$ & $1-2$ & $1.5 \pm 0.52$ & 133.6 & $<0.0001^{*}$ \\
\hline PSH & $10-40$ & $23.3 \pm 11.2$ & $5-13$ & $8.8 \pm 2.48$ & $5-10$ & $6.7 \pm 1.49$ & 18.46 & $<0.0001^{*}$ \\
\hline Postural tachycardia & $13-39$ & $23.8 \pm 8.03$ & $13-26$ & $14.3 \pm 4.5$ & $11-21$ & $15.3 \pm 2.98$ & 6.31 & $0.0044^{*}$ \\
\hline $\mathrm{HRDB}$ & $5-16$ & $10.7 \pm 3.4$ & $9-18$ & $13.1 \pm 2.76$ & $9-18$ & $13.9 \pm 2.88$ & 4.08 & $0.025^{*}$ \\
\hline HRV & $1.1-1.3$ & $1.25 \pm 0.06$ & $1.3-1.4$ & $1.29 \pm 0.04$ & $1.2-1.4$ & $1.29 \pm 0.04$ & 3.40 & 0.51 \\
\hline
\end{tabular}

$H R D B$ heart rate response to deep breath, HRV heart rate response to Valsalva, NDS neuropathy disability score, NSS neuropathy symptom score, $P S H$ postural systolic hypotension

*Significant 
Table 2 Sleep parameters among diabetic neuropathy patients (group I), diabetic patients without neuropathy (group II) and healthy control (group III)

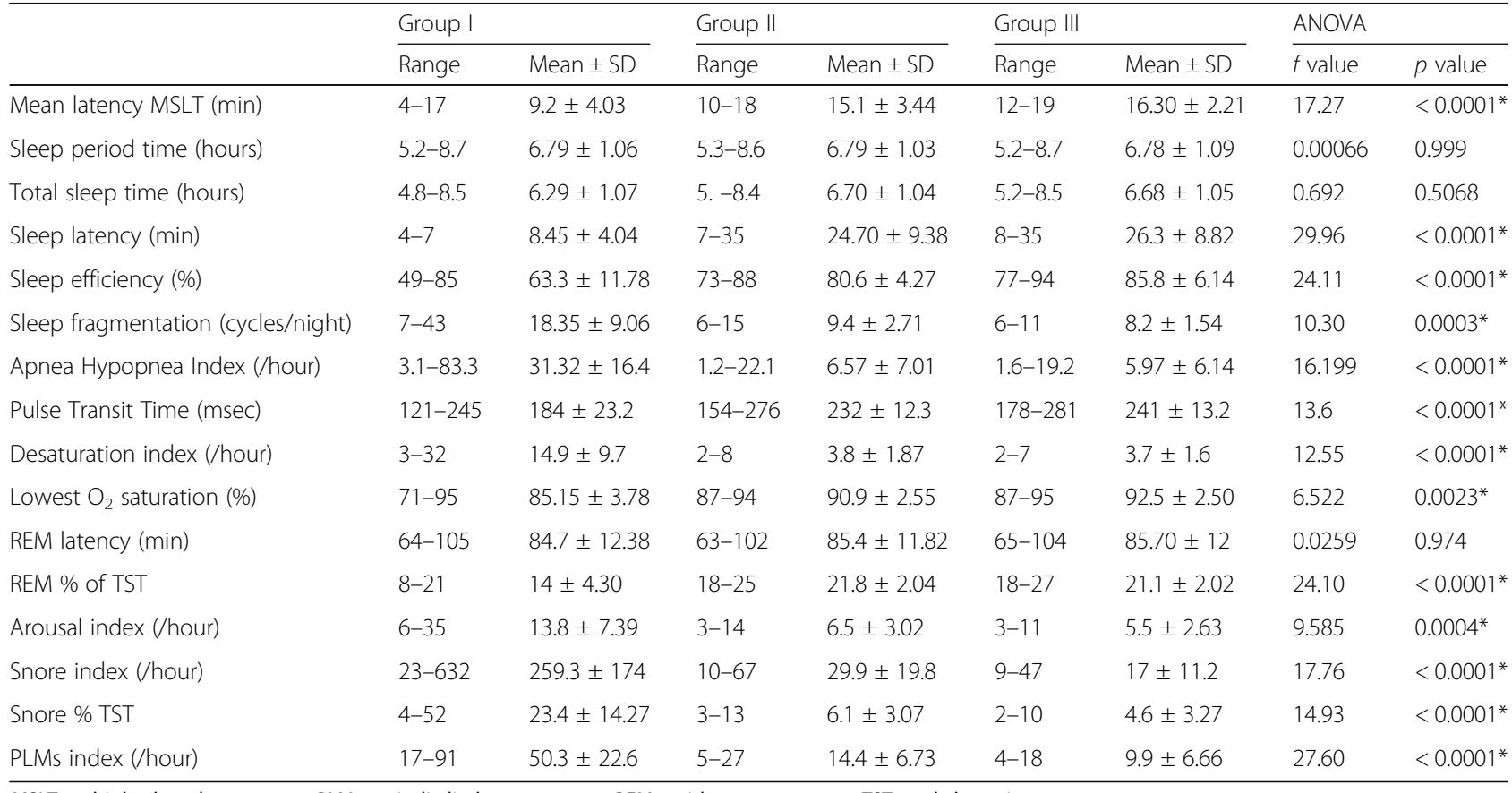

MSLT multiple sleep latency test, PLMs periodic limb movements, REM rapid eye movement, TST total sleep time

*Significant

due to sympathetic overdrive, increased accidents due to decreased attention, cognitive decline, and mood changes (Aurora and Punjabi 2013). Sleep disordered breathing is a common yet under-researched diabetic neuropathy complication with consequent decreased patients' productivity, increased insulin resistance, and impaired glycemic control (Nannapaneni et al. 2013).

This study showed marked increase in the incidence of sleep disordered breathing in DPN patients (40\%) than diabetic patients without neuropathy and healthy control subjects. These results are in accordance with the work of Moon and her colleague (Moon et al. 2015) who found SAS in 50\% of their DPN studied patients and attributed this high incidence to impaired bulbar function, reduced pharyngeal tone, diaphragmatic weakness, and even a central component due to impaired chemoreceptors functions. The relatively higher incidence than the present study may be attributed to higher BMI and ages among their studied patients. The last clarification of reduced chemoreceptors functions may be an explanation of the 2 DPN patients who showed mixed obstructive and central sleep apnea in this study.

On the other hand, Meng and his colleagues (Meng et al. 2016) found no relation between the existence of neuropathy in diabetic patients and the presence of sleep complaints. This different result may be explained by their dependence on a subjective sleep quality questionnaire (difficulty falling asleep, early final awakening, short or long sleep) without the use of objective PSG and MSLT. From this difference, we can conclude that many DPN patients may not be aware of their disordered sleep breathing which may only cause frequent micro-arousals not sufficient to awaken the patient. These micro-arousals may not affect the total sleep time

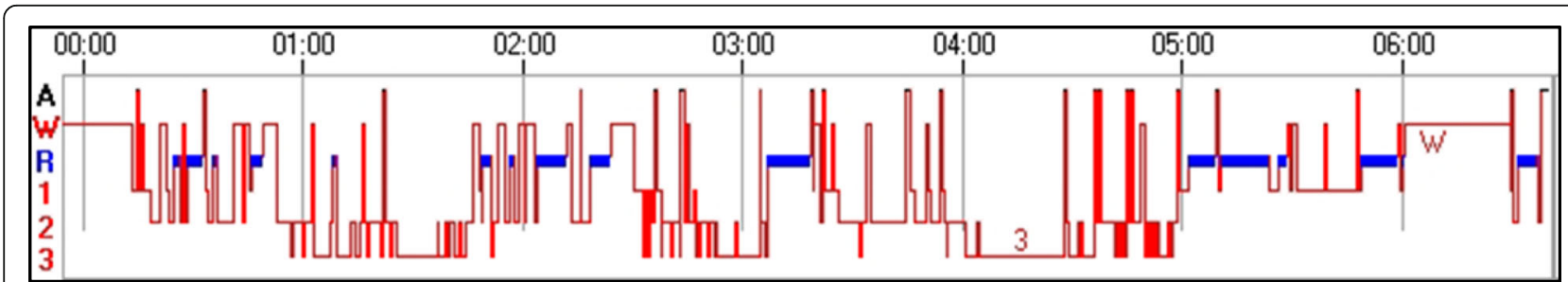

Fig. 1 Hypnogram of a diabetic patient with peripheral neuropathy showing sleep fragmentation and increase in each of waking after sleep onset, arousal index, and sleep stage transition index 
Table 3 Correlations of the multiple sleep latencies and apnea hypopnea indices with other variables in diabetic peripheral neuropathy patients

\begin{tabular}{|c|c|c|c|c|}
\hline & \multicolumn{2}{|l|}{ MSLT } & \multicolumn{2}{|c|}{ Apnea hypopnea index } \\
\hline & $r$ & $\mathrm{p}$ & $r$ & $p$ \\
\hline Disease duration & -0.561 & $0.0018^{*}$ & +0.703 & $0.0005^{*}$ \\
\hline HBA1C (\%) & -0.663 & $0.0014^{*}$ & +0.868 & $<0.0001^{*}$ \\
\hline Neuropathy symptom score & -0.812 & $<0.0001^{*}$ & +0.428 & $<0.0001^{*}$ \\
\hline Neuropathy disability score & -0.669 & $0.0012^{*}$ & +0.806 & $<0.0001^{*}$ \\
\hline Postural systolic hypotension & -0.796 & $<0.0001^{*}$ & +0.843 & $<0.0001^{*}$ \\
\hline Postural tachycardia & -0.474 & 0.0344 & +0.843 & $<0.0001^{*}$ \\
\hline
\end{tabular}

MSLT Multiple sleep latency test

*Significant

but disturb the sleep quality with its diurnal consequences in the form of EDS, cognitive impairment, and mood changes.

The present study had elucidated that most of sleep disordered breathing in DPN was OSA which was evidenced by the high respiratory effort and the significant diminution in pulse transit time during the apnea period. These results agree with that of Nagayoshi and his colleagues (Nagayoshi et al. 2016) and Rasche and colleague (Rasche et al. 2010) who found that OSA is the predominant sleep respiratory disturbance in their studied DPN patients than healthy control subjects, but the incidence of SAS was lower in their works $(25 \%$ of DPN patients). This difference might be due to the younger ages, shorter disease durations and lower BMI in their included patients which significantly influence the rate of SAS irrespective to the existence of DPN.

The present study declared that, PSG abnormalities are not only higher among DPN patients but also proportional with the severity of sensori-motor manifestations as measured by the NSS and NDS. These data are in accordance with that of Tahrani and his colleagues (Tahrani et al. 2012) and Oleinikov and Sergatskaya (Oleinikov and Sergatskaya 2012). At the same time, they concluded that tight diabetic control cannot be achieved without correction of the OSA by CPAP (continuous positive airway pressure ventilation).

The present study showed that most of DPN patients with sleep disordered breathing had clinical manifestations of dysautonomia either in their complaints (postural dizziness, urinary symptoms, erectile dysfunctions, gustatory sweating, and gastrointestinal symptoms) and/ or in examinations (postural systolic hypotension, postural tachycardia, and abnormal heart rate response to deep breath). These results are keeping with each of Dimitropoulos and his colleagues (Dimitropoulos et al. 2014) and Rasche and his colleagues (Rasche et al. 2013) who found a high rate of OSA among DPN patients who had dysautonomia especially that affecting the cardiac parasympathetic and/or sympathetic supply.

At the same time, PSG studying of DPN patients revealed marked impairment of their sleep architecture beyond the sleep apnea in the form of decreased mean latencies of MSLT and sleep efficiencies with markedly increased sleep fragmentations, snore, and periodic limb movements' indices which may result in impaired sleep quality beyond the diminished sleep time caused by increased walking after sleep onset and repeated arousals. These results were in accordance with that of Cerón and his colleagues (Cerón et al. 2015) and Reutrakul and
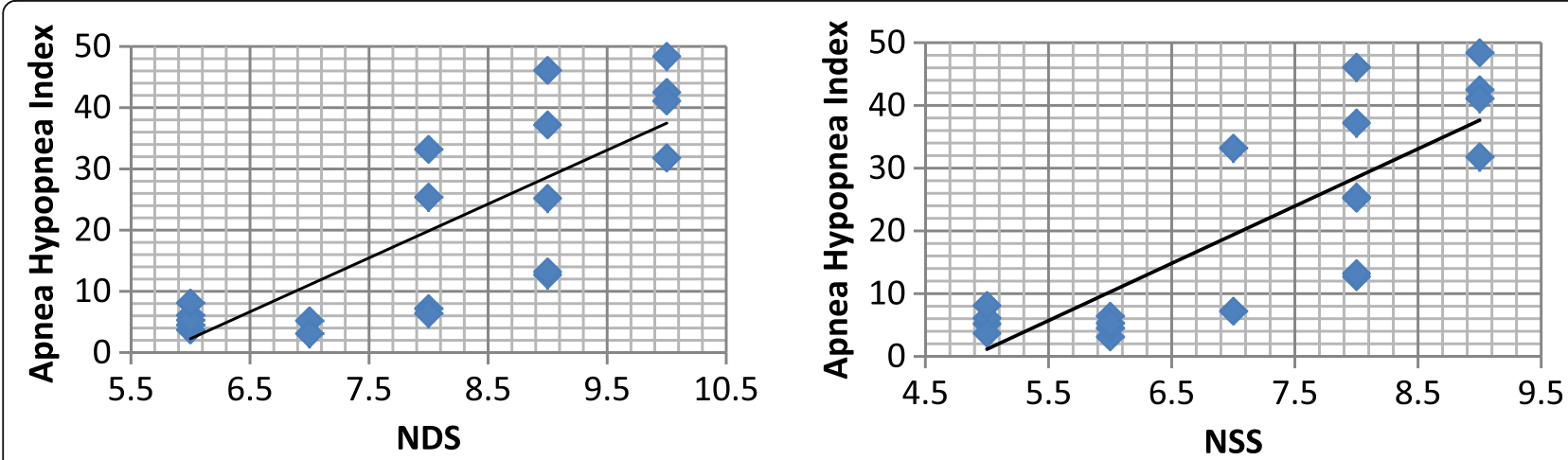

Fig. 2 Positive correlation between apnea hypopnea index and each of neuropathy disability score (left) and neuropathy symptom score (right) in diabetic peripheral neuropathy patients 
Mokhlesi (Reutrakul and Mokhlesi 2017) who found a bi-directional relationship between DPN and sleep architecture. The decreased sleep efficiency and the increased sleep fragmentation beside the SAS causes activation of the hypothlamo-pituitary-adrenal axis leading to increased sympathetic overactivity, oxidative stress, and increased systemic inflammation which in turn result in metabolic dysfunction and higher insulin resistance.

\section{Conclusions}

Type 2 diabetic patients complicated by peripheral neuropathy are at increased risk of developing sleep disturbances mainly obstructive sleep apnea, decreased sleep efficiency, sleep fragmentation, and frequent nocturnal hypoxia. These sleep abnormalities significantly disturb patient's diurnal activities leading to excessive daytime sleepiness, decreased productivity, impaired cognition, mood disturbances, higher rate of accidents, increased insulin resistance, and poor glycemic control. So, early diagnosis and management of sleep disturbances in DPN patients is very important for holistic patient's management, to increase his productivity, reduce the rate of his diabetic complications, and improve his quality of life.

\section{Abbreviations \\ AHI: Apnea hypopnea index; DAN: Diabetic autonomic neuropathy; DM: Diabetes mellitus; DNF: Diabetic neuropathies free; DPN: Diabetic peripheral neuropathy; EDS: Excessive daytime sleepiness; MSA: Mixed sleep apnea; MSLT: Multiple sleep latency test; NDS: Neuropathy disability score; NSS: Neuropathy symptom score; OSA: Obstructive sleep apnea; PSG: Polysomnogram; REM: Rapid eye movement; SAS: Sleep apnea syndromes; TST: Total sleep time}

\section{Acknowledgements}

We would like to thank the team of the Neuropsychiatry and Chest Departments, Faculty of Medicine, Tanta University, for their help in case selection and polysomnogram processing.

\section{Consent of publication}

All participants had signed an informed consent to participate and for the data to be published.

\section{Availability of data and materials}

The datasets used and/or analyzed during the current study are available from the corresponding author on reasonable request and could be publicly available.

\section{Authors' contributions}

WSB participated in the study idea, design, patients' collection, raw data recording and statistical analysis, PSG and MSLT interpretation, references collection, and manuscript writing and revision. YAEH participated in the study design, patients' collection, data and statistical analysis, and manuscript writing and revision. EASE participated in patients' collection, statistical analysis, and manuscript writing and revision. NAAL participated in patients' evaluation and assessment (clinical neuropathy and autonomic nervous system assessment) and references and data collection. ISEl participated in patients' and control chest evaluation, study design, MSLT and PSG interpretation, and manuscript revision. All authors read and approved the final manuscript.

\section{Ethics approval and consent to participate}

The manuscript was approved by The Research Ethics Committee and Quality Assurance Unit, Faculty of Medicine, Tanta University.

- The URL: http://tqac.tanta.edu.eg/new-tqac/
http://QualityAssuranceUnit@hotmail.com

- Approval Code: 30600/11/15

- Name of the PI: Wafik Said Kamel ElBahnasy.

- Name of the department: Neuropsychiatry.

- Type of the research: promotion research.

- Date of approval: November 2015.

- The protocol of the study was approved by The Research Ethics Committee and Quality Assurance Unit, Faculty of Medicine, Tanta University. Participation was voluntary, all participants received detailed information concerning the aims of the study and the possible risks, and an informed consent was obtained from all prior to the commencement in the study.

\section{Competing interests}

The authors declare that they have no competing interests.

\section{Publisher's Note}

Springer Nature remains neutral with regard to jurisdictional claims in published maps and institutional affiliations.

\section{Author details}

${ }^{1}$ Department of Neuropsychiatry, Tanta University, Tanta 31527, Egypt. ${ }^{2}$ Department of Chest Diseases, Tanta University, Tanta 31527, Egypt.

${ }^{3}$ Department of Neuropsychiatry, Faculty of Medicine, Tanta University, Tanta 31511, Egypt.

Received: 4 October 2017 Accepted: 26 August 2018

Published online: 04 September 2018

\section{References}

Aurora RN, Punjabi NM. Obstructive sleep apnoea and type 2 diabetes mellitus: a bidirectional association. Lancet Respir Med. 2013;1(4):329-38. https://doi.org/ 10.1016/S2213-2600(13)70039-0.

Cabezas-Cerrato J. The prevalence of clinical diabetic polyneuropathy in Spain: a study in primary care and hospital clinic groups. Neuropathy Spanish Study Group of the Spanish Diabetes Society. Diabetologia. 1998;41(11):1263-9.

Cerón EM, Mateos RC, García-Río F. Sleep apnea-hypopnea syndrome and type 2 diabetes. A reciprocal relationship. Arch Bronconeumol. 2015;51(3):128-39. https://doi.org/10.1016/j.arbr.2014.12.007.

Dimitropoulos G, Tahrani AA, Stevens MJ. Cardiac autonomic neuropathy in patients with diabetes mellitus. World J Diabetes. 2014;5(1):17-39. https://doi. org/10.4239/wjd.v5.i1.17.

Feldman EL, Nave KA, Jensen TS, Bennett DLH. New Horizons in Diabetic Neuropathy: Mechanisms, Bioenergetics, and Pain. Neuron. 2017;93(6):1296-313. https://doi.org/10.1016/j.neuron.2017.02.005.

Grigg-Damberger MM. The AASM Scoring Manual four years later. J Clin Sleep Med. 2012:8(3):323-32. https://doi.org/10.5664/jcsm.1928.

Kisozi T, Mutebi E, Kisekka M, Lhatoo S, Sajatovic M, Kaddumukasa M, et al. Prevalence, severity and factors associated with peripheral neuropathy among newly diagnosed diabetic patients attending Mulago hospital: a cross-sectional study. Afr Health Sci. 2017;17(2):463-73. https://doi.org/10. 4314/ahs.v17i2.21.

Littner MR, Kushida C, Wise M, Davila DG, Morgenthaler T, Lee Chiong T, et al. Practice parameters for clinical use of the multiple sleep latency test and the maintenance of wakefulness test. Sleep. 2005;28(1):113-21.

Martin CL, Albers JW, Pop-Busui R. Neuropathy and related findings in the diabetes control and complications trial/epidemiology of diabetes interventions and complications study. Diabetes Care. 2014;37:31-8. https:// doi.org/10.2337/dc13-2114

Meng LL, Liu Y, Geng RN, Tang YZ, Li DQ. Association of diabetic vascular complications with poor sleep complaints. Diabetol Metab Syndr. 2016;8:80. https://doi.org/10.1186/s13098-016-0195-8.

Moon K, Punjabi NM, Aurora RN. Obstructive sleep apnea and type 2 diabetes in older adults. Clin Geriatr Med. 2015;31:139-47. https://doi.org/10.1016/j.cger. 2014.08.023.

Nagayoshi M, Punjabi NM, Selvin E, Pankow JS, Shahar E, Iso H, et al. Obstructive sleep apnea and incident type 2 diabetes. Sleep Med. 2016;25:156-61. https://doi.org/10.1016/j.sleep. 2016.05.009.

Nannapaneni S, Ramar K, Surani S. Effect of obstructive sleep apnea on type 2 diabetes mellitus: a comprehensive literature review. World J Diabetes. 2013; 4(6):238-44. https://doi.org/10.4239/wjd.v4.i6.238. 
Oleinikov VE, Sergatskaya NV. CPAP therapy for obstructive sleep apnea syndrome in patients with carbohydrate metabolic disturbances and type 2 diabetes mellitus. Ter Arkh. 2012;84(11):94-8.

Polonsky KS, Burant CF. Type 2 Diabetes Mellitus. In: Melmed S, Polonsky KS, Larsen PR, Kronenberg HM, editors. Williams textbook of endocrinology, vol. 31. 13th ed. Canada: Elsevier; 2016. p. 1386-450.

Pop-Busui R, Evans GW, Gerstein HC, Fonseca V, Fleg JL, Hoogwerf BJ, et al. Effects of cardiac autonomic dysfunction on mortality risk in the Action to Control Cardiovascular Risk in Diabetes (ACCORD) trial. Diabetes Care. 2010; 33:1578-84. https://doi.org/10.2337/dc10-0125.

Qu GB, Wang LL, Tang X, Wu W, Sun YH. The association between vitamin D level and diabetic peripheral neuropathy in patients with type 2 diabetes mellitus: An update systematic review and meta-analysis. J C Trans Endocrinol. 2017;9:25-31. https://doi.org/10.1016/j.jcte.2017.04.001

Rasche K, Keller T, Tautz B, Hader C, Hergenç G, Antosiewicz J, et al. Obstructive sleep apnea and type 2 diabetes. Eur J Med Res. 2010;15(II):152-6. https://doi. org/10.1186/2047-783X-15-S2-152

Rasche K, Keller T, Hader C, Leidag M, Prinz C. Impact of Obstructive Sleep Apnea on Type 2 Diabetes and Vice Versa. European Endocrinology. 2013;9(2):107-9. https://doi.org/10.17925/EE.2013.09.02.107.

Reutrakul S, Mokhlesi B. Obstructive sleep apnea and diabetes: a state of the art review. Chest. 2017;152(5):1070-86. https://doi.org/10.1016/..chest.2017.05.009.

Sateia MJ. International classification of sleep disorders, third edition, highlights and modifications. Chest. 2014;146(5):1387-94. https://doi.org/10.1378/chest. 14-0970.

Schober AK, Neurath MF, Harsch IA. Prevalence of sleep apnoea in diabetic patients. Clin Respir J. 2011;5(3):165-72. https://doi.org/10.1111/j.1752-699X. 2010.00216.x.

Tahrani A, Ali A, Neil TR, Safia B, Kiran D, Shanaz M, et al. Obstructive sleep apnea and diabetic neuropathy. A novel association in patients with type 2 diabetes. Am J Respir Crit Care Med. 2012;186(5):434-41. https://doi.org/10. 1164/rccm.201112-21350C.

Villafaina S, Collado-Mateo D, Fuentes JP, Merellano-Navarro E, Gusi N. Physical exercise improves heart rate variability in patients with type 2 diabetes: a systematic review. Curr Diab Rep. 2017:17(11):110. https://doi.org/10.1007/ s11892-017-0941-9.

\section{Submit your manuscript to a SpringerOpen ${ }^{\circ}$ journal and benefit from:}

- Convenient online submission

- Rigorous peer review

- Open access: articles freely available online

- High visibility within the field

- Retaining the copyright to your article

Submit your next manuscript at $\boldsymbol{\nabla}$ springeropen.com 